\title{
Word importance-based similarity of documents metric (WISDM)
}

\author{
[Fast and scalable document similarity metric for analysis of scientific documents] \\ Viktor Botev \\ IRIS.AI \\ Bekkestua, Norway \\ victor@iris.ai \\ Kaloyan Marinov \\ IRIS.AI \\ Bekkestua, Norway \\ kaloyan.marinov@iris.ai \\ Florian Schäfer \\ IRIS.AI \\ Bekkestua, Norway \\ florian.joh.schaefer@gmail.com
}

\begin{abstract}
We present the Word importance-based similarity of documents metric (WISDM), a fast and scalable novel method for document similarity/distance computation for analysis of scientific documents. It is based on recent advancements in the area of word embeddings. WISDM combines learned word vectors together with traditional count-based models for document similarity computation, eventually achieving state-of-the-art performance and precision. The novel method first selects from two text documents those words that carry the most information and forms a word set for each document respectively. Then it relies on an existing word embeddings model to get the vector representations of the selected words. In the final step, it computes the closeness of the two sets of word vector representations, fit into a matrix, using a correlation coefficient. The presented metric was evaluated on three tasks, relevant to the analysis of scientific documents, and three data sets of open access scientific research. The results demonstrate that WISDM achieves significant performance speed-up in comparison to state-of-the-art metrics with a very marginal drop in precision.
\end{abstract}

\section{CCS CONCEPTS}

- Computing methodologies $\rightarrow$ Information extraction; Lexical semantics; Language resources;

\section{KEYWORDS}

document similarity, document distance, text metric, document metric, word embeddings, similarity, correlation coefficient, importancebased, TF-IDF, word2vec

\section{ACM Reference format:}

Viktor Botev, Kaloyan Marinov, and Florian Schäfer. 2017. Word importancebased similarity of documents metric (WISDM). In Proceedings of WOSP 2017, Toronto, ON, Canada, fune 19, 2017, 7 pages.

https://doi.org/10.1145/3127526.3127530
Permission to make digital or hard copies of part or all of this work for personal or classroom use is granted without fee provided that copies are not made or distributed for profit or commercial advantage and that copies bear this notice and the full citation on the first page. Copyrights for third-party components of this work must be honored. For all other uses, contact the owner/author(s).

WOSP 2017, Fune 19, 2017, Toronto, ON, Canada

(c) 2017 Copyright held by the owner/author(s).

ACM ISBN 978-1-4503-5388-5/17/06.

https://doi.org/10.1145/3127526.3127530

\section{INTRODUCTION}

The number of research papers published on a daily basis today is unprecedented. Just within one field such as machine learning more than $1 \mathrm{M}$ papers have been published to date and around 40000 new papers are published every year. (A search with "machine learning" on the CORE research database [18] yields 1004747 articles - last accessed 2017-04-28 - out of which about 40000 are from 2016 and that is just open access content.) As a consequence, researchers can at best keep track of a rather small sub-domain by continuously following that area. This makes it hard for people new to the field to get a general understanding of the topic based on the most eminent research results. An even bigger challenge arises when trying to connect new findings across area borders.

To tackle this problem, researchers in the fields of natural language processing (NLP) and information retrieval (IR) have been working for several years on generating methods for analysis of documents, potentially enabling researchers to gather the most relevant tens to hundreds of documents. This analysis includes solving not only problems such as Nearest Neighbour Search (NNS), but also topic prediction, document distance calculation, filtering tasks (classification of documents as relevant/not relevant), etc. This is needed, because in many cases users need guidance to the end result as they do not know from the start which exact parameters to look for. All of these tasks can be addressed with computation of a similarity or distance metric between documents.

A usual first step in computing textual similarities is building a representation of the text in some mathematical form and then, based on this representation, computing the distance or similarity between the documents. Depending on the type of textual representation we can distinguish between three types of document similarity methods existing in the literature - count-based, probabilistic and embeddings-based. Traditionally, textual representations are created using count-based methods such as TF-IDF [17], or Latent Dirichlet Allocation (LDA) [5]. More recently neural word embeddings have become the state-of-the-art, where representations are learned by a neural network $[4,13]$. As presented in the literature, these neural representations perform generally better on a wide range of tasks compared to count-based methods [3]. The countbased methods are usually simpler than the rest and require less computational resources, but are also less accurate in terms of precision. Probabilistic approaches are mainly targeting classification tasks or topic predictions. That is why they are not very well suited for as large variety of tasks as required for narrowing down the existing scientific literature to the few documents addressing the researcher's problem. Finally the embeddings-based metrics are more 
complex, require training time, and more computational resources, but yield much better results [2,11].

Another categorization of existing methods is based on their reliance on human supervision (supervised) or on learning the representation just by reading the text (unsupervised). Supervised methods are based on a definitive ground truth, meaning that for each document (maybe sometimes for each embedding) there has to be a label associated with it. In the ambiguous context of humangenerated text, this manual labelling inevitably introduces some degree of subjectivity. Additionally, the creation of larger evaluation sets may result in a considerable and often even unfeasible amount of work. For a corpus the size of $70 \mathrm{M}+$ documents [18], it is required that at least 3.5 million documents be labelled with their respective ground truth. This compromises the scalability of such metrics, essentially making them not feasible for large data sets such as the ones discussed. Nevertheless they can be a vital part of evaluating the quality of an unsupervised metric and play an essential role in their development.

In this work we present the Word importance-based similarity of documents metric (WISDM). WISDM is an embed-dings-based method that benefits from the simplicity and efficiency of a TFIDF model to optimize the document similarity computation by keeping the quality of the results on par with the state-of-theart embeddings methods, while showing significant performance improvements and memory usage reduction. Although WISDM can take advantage of human-curated labels and in this way become a supervised method, this work will focus on its unsupervised version and leave its supervised extension for future work. The evaluation of the method is focused on measuring precision and efficiency. It applies as a baseline two embeddings-based state-of-the-art metrics, namely SIF[2] and WMD[11] together with WISDM on an NNS task, on a topic prediction task, and on a filtering task. As main data set, a subset from the CORE database [18] with about $1.2 \mathrm{M}$ document abstracts is used. Additionally two subsets of the main data set are formed containing human-assigned labels generated by the Iris.ai AI training community.

The rest of the paper is organized as follows: Section 2 discusses the state-of-the-art and the selection of the two baseline metrics. In Section 3 we present the WISDM in more details. Section 4 describes the evaluation framework, the tasks, and the results for the conducted experiments. Finally, Section 5 concludes the paper.

\section{RELATED WORK}

In the following section we will present the related work around the different categories of document similarity methods and their relation to the current work.

Probabilistic methods such as perplexity measure are some of the oldest methods for evaluating language models. They have been widely used for years although studies show that the correlation between perplexity and human judgment is quite low [7]. The reason is that this unsupervised quantity can easily be computed for a given word sequence. In formal terms it is defined (cf. [6]) as the inverse probability of the given word sequence occurring in the test set, normalized by the number of words. More specifically, the perplexity of the word sequence $w_{1} \ldots w_{N}$ is defined as:

$$
P P\left(w_{1} \ldots w_{N}\right)=p\left(w_{1} \ldots w_{N}\right)^{-\frac{1}{N}}
$$

In recent years, LDA [5] has become a widely used method for topic prediction and modelling. However, it has not produced significant results in terms of document distance computation, which is one of our target tasks.

Count-based methods such as TF-IDF continue being popular for the same reason - because of their simplicity and computational efficiency. However they are usually not used as a standalone method, because their results do not achieve high enough precision. Instead they are applied in combination with others as presented in the paper [2] by Arora et al. WISDM is inspired by these methods and is designed to take advantage of the TF-IDF scheme to reduce the amount of computations necessary for computing a document similarity.

Nowadays, neural network based methods for computing word embeddings have become the basis for several methods addressing the task of computing distances between pieces of text (sentences, paragraphs, and beyond) [12]. Two particular methods show stateof-the-art results outperforming both count-based and probabilitybased methods and also other neural network based methods when used for sentence/ paragraph/ text similarity computations - Word Mover's Distance (WMD) [11] and Smooth Inverse Frequency (SIF) [2].

The first method, WMD, is an unsupervised method for computing the distance between text documents. This distance function relies on the availability of word embeddings and an (unlabeled) corpus $C$ of documents. On a high level, the WMD between documents $d_{1}, d_{2}$ quantifies the minimum distance that the words of $d_{1}$ need to "travel in order to be transformed into" the words of $d_{2}$. This approach is rather complex and requires significant computational resources as noted by the authors, especially in the case of long texts and a corpus vocabulary containing a large number of unique words. Unfortunately in the scientific context we both have large texts and vocabulary richer than in many other cases (for example news or general English). The reason to mention WMD in this section though is that it shows very good results in terms of precision compared to other methods. Furthermore it has a supervised extension (S-WMD) [10] that allows the WMD to take advantage of labelled data if available. In that regard, WISDM is inspired by WMD and designed to accommodate supervised data as well.

The authors of the second method, SIF, developed an unsupervised algorithm which learns high-quality vector embeddings of sentences from a given corpus. The algorithm relies on the availability of word embeddings (computed via word2vec [13] or GloVe [14] on an unlabeled corpus such as Wikipedia), an (unlabeled) corpus $C$ of sentences, and (estimated) frequencies $p(w)$ for each word $w$ in $C$ 's vocabulary. The word frequencies $p(w)$ are used to define smooth inverse frequencies (SIFs). The sentence vectors are built by computing first a SIF-weighted average of the word vectors, and subsequently subjected to a modification based on PCA/SVD. If a corpus of documents (instead of sentences) is available, this same scheme can be applied in order to learn vector representations for the corpus documents. This approach is significantly simpler than WMD and achieves similar precision results with less computational resources, which makes it a tough to beat baseline for document distance computation as stated by the authors.

The current work is inspired mainly by the last two approaches, but is focused on computational efficiency by taking advantage of 
the simplicity and efficiency of the count-based TF-IDF model. The idea is to maintain on par precision with WMD and SIF and at the same time reduce the computational time and memory consumption. This will enable development of tools on top of it that can help researchers operate with corpora of tens of millions documents and be able to solve NNS tasks, topic prediction tasks and other filtering tasks within a reasonable amount of time without the necessity of having a super computer.

\section{METHOD}

The current section has the purpose of objectively presenting the WISDM method for computing document similarity.

\subsection{Overview}

The Word importance-based similarity of documents metric is constructed out of two major components - the first component is a TF-IDF model, and the second component is a word2vec model. The TF-IDF model is used to score the words/phrases in the document and select the keywords/key-phrases (key-tokens ${ }^{1}$ ). The final document representation is a matrix of the document's key-tokens' representations, where each row in the matrix is the word2vec embedding of the corresponding key-token. To compute the distance between two documents $d_{1}$ and $d_{2}$ WISDM uses the RV coefficient [16] between the document representations of the corresponding documents.

\subsection{Mathematical and algorithmic framework}

Let us first give a high level explanation of the key-tokens selection. When given a text document $d$, it is first tokenized into word/phrase tokens, then each token is scored by TF-IDF and is thus assigned a token importance score. After that, all the tokens with an importance score above a certain threshold $\theta$ (key-tokens) are selected to represent the document.

Suppose now that $d_{1}, d_{2}$ are two given documents. For $i=1,2$, let $w_{1}^{i}, \ldots, w_{n_{i}}^{i}$ denote the key-tokens in $d_{i}$ and assume they are all covered by the available word2vec model.

The WISDM measure of similarity between $d_{1}, d_{2}$ is computed as follows:

(1) Form document matrix.

For $j=1,2$, let $v_{1}^{j}, \ldots, v_{n_{j}}^{j}$ be the ( $k$-dimensional) word2-vec embeddings corresponding to $w_{1}^{j}, \ldots, w_{n_{j}}^{j}$, and introduce the following document representative matrices:

$$
V_{j}:=\left[\begin{array}{ccc}
\mid & & \mid \\
v_{1}^{j} & \ldots & v_{n_{j}}^{j} \\
\mid & & \mid
\end{array}\right] \in \mathbb{R}^{k \times n_{j}} \text { for } j=1,2
$$

(2) Compute document similarity.

Compute the similarity between $d_{1}, d_{2}$ by computing the RV coefficient

$$
\operatorname{RV}(X, Y):=\frac{Y^{T} X \cdot Y^{T} X}{\sqrt{X^{T} X \cdot X^{T} X} \sqrt{Y^{T} Y \cdot Y^{T} Y}} .
$$

where, if $A, B$ are two matrices with the same shape, then $A \cdot B:=\sum_{i, j} a_{i, j} b_{i, j}$

${ }^{1}$ Key-tokens could be any sequence of $\mathrm{n}$-grams for which a vector representation exists. For the current work key-tokens are limited only to uni-grams and bi-grams.

\section{EVALUATION}

This section presents the framework for comparing WISDM against the (selected) baseline methods and the final results. The section starts with a presentation of the evaluation tasks and measures. Next the data sets are described and the experimental setup is formalized. Finally, the section is concluded with the results.

\subsection{Evaluation tasks and measures}

After a model for distance computation between two documents has been trained, we need to test and evaluate its performance and accuracy according to the three tasks defined in Section 1. In order to evaluate the quality of the method on a test document corpus $C_{t}$ and be able to draw conclusions, we will use supervised metrics specifically adapted for each task. We will describe each task setting and labeling information within the corresponding task definition.

The first task is topic prediction (TTP). It has the goal of predicticting a topic label for a given document $d$. Our supervised metric $l$ will have a predefined label for each document as the ground truth. In more formal terms:

- Suppose the labeling information on $C_{t}$ is available in the form of classification, i.e. in the form of a function $l$ on $C_{t}$ which classifies each $d \in C_{t}$ as belonging to exactly one of finitely many classes. Then, the distance-computing mechanism and a $k \mathrm{NN}$ majority decision rule [8] is used to predict a class for each $d \in C_{t}$. Ultimately, we wish to compute the agreement between the ground-truth class labels from $l$ and the predicted class labels for all documents in $C_{t}$.

The second task is document filtering (TF), which has the goal of deciding whether a document $d_{1}$ is relevant or not to a base document $d$. The supervised metric $r n r(d)$ assigns a binary label to each document in $C_{t}$ based on its relevance to $d$. In more formal terms:

- Suppose the labeling information on $C_{t}$ is available in the form of the following two sets for each document $d: D^{+}(d)$ of all relevant and $D^{-}(d)$ of all not relevant documents to $d$. Then, we can check if the trained distance-computing mechanism yields smaller distances between $d, d^{+}$than between $d, d^{-}$for all $d^{+} \in D^{+}(d)$ and $d^{-} \in D^{-}(d)$. Another possible verification could be to check whether all documents within certain distance $\lambda$ are in $D^{+}(d)$ and whether all documents with distance bigger than $\lambda$ are in $D^{-}(d)$.

Finally, the third task (TKNN) aims to find the $k$-nearest neighbours $(k \mathrm{NN})$ of a document $d$. Generating a supervised metric, where each document is labelled with its $k$-nearest neighbours, even for smaller ( $k=2$ or 3 ) is rather difficult and not scalable. Instead we used a human-labelled data set, where each document is annotated with keywords based on human judgment. We utilize those keywords to define a true distance between $d_{1}$ and $d_{2}$. At the end we expect that the $k \mathrm{NN}$ found by the metric under evaluation will overlap with the $k \mathrm{NN}$ identified by the true distance metric. In more formal terms:

- Suppose the labeling information on $C_{t}$ is available in the form of human-provided keywords $\left\{w_{j}^{d}\right\}_{j=1}^{i}$ for each $d \in C_{t}$. We can construct a vector representation $v \in \mathbb{R}^{M}$ of each $d \in C_{t}$ based on the keywords, where the vector's size $M$ 
Table 1: Data sets for evaluation.

\begin{tabular}{lrcll}
\hline Data set & Num of docs & Avg num of words per doc & Labels & Task \\
\hline DLarge & 1119687 & 250 & Topic label $(100$ possible topics $)$ & TTP \\
Drnr & 2217 & 312 & Two sets $D^{+}(d), D^{-}(d)$ & TF \\
DKey & 1400 & 283 & Set of keywords & TKNN \\
DTrain & 4590402 & 298 & - & - \\
\hline
\end{tabular}

is the number of unique words in the vocabulary of the annotations. The entries of $v$ are 0 or 1 based on whether the corresponding word is a keyword in $d$ or not. This can subsequently be used to compute the distance between two documents using the vectors' cosine distance. At the end we compute the distances for the $k \mathrm{NN}$ found by the metric under examination for $d$ and we expect the so defined true distance to produce overlapping results.

After presenting the metrics and measures we used in the experimental setup, let us look at the data sets on which we applied those metrics.

\subsection{Data sets}

For the purpose of evaluating WISDM we will be using three different subsets of the CORE database which have been labelled as formally described in Section 4.1. The properties and sizes of the data sets are presented in Table 1. Each of the four data sets consists of a number of paper abstracts as per the Num of docs column. An average document length for each set is shown in the next column. The last column marks the task for which the data set is used. The fourth data set, listed in Table 1, is used for training the word2vec model which is the foundation for all three methods under evaluation. As presented it is unlabelled and contains $4.5 \mathrm{M}+$ documents.

The next subsection will present how these data sets are used together with the defined metrics in Section 4.1 .

\subsection{Experimental setup}

In this section we will describe first the software and hardware setup for evaluation and then the experimental setup per task.

All experiments were conducted on an Amazon AWS EC2 instance of type m4.4xlarge [1]. Essentially a $2.4 \mathrm{GHz}$ Intel Xeon E5-2676 v3 (Haswell) physical CPU with allocated 16 vCPUs for the machine and 64GB of RAM. However, when running an experiment we used the taskset Linux command to limit the execution on just a single CPU. For performance measure we use CPU clock and wall time and for memory usage we use the VmSize property of a Linux process. In order to ensure a setup as close as possible, all three models share the same word2vec model, trained on DTrain with a vocabulary that consists of 415457 unique word tokens.

For the software setup and further experiments we had to select a value for the only hyper-parameter $\theta$ of WISDM. Figure 1 shows the relation between $\theta$ and the average number of words per document selected by the method's TF-IDF component. The fewer number of words are selected the better the performance becomes. On the other hand selecting more words is supposed to increase the precision, but for our experiments selecting $\theta<0.1$ leads to very little or no improvement to the precision and only degrades the execution time. Therefore we selected $\theta=0.1$ for the conducted experiments. Figure 1 illustrates that this selection result in an average of 35 selected words per document. Given the average length of documents from Table 1 that means only between $11 \%$ to $14 \%$ of all words are kept for comparison. In order to illustrate how changing $\theta$ affects processing time and precision, in Appendix A we attach results from experiments with different values for $\theta$ on the document filtering task. Although those results give perspective, the tuning procedure, that allows selection of one value of $\theta$, which performs well on a variety of tasks, is not the focus of this work, but is left for future work instead.

Each task has a different setup based on the respective data set and precision measures. For the TTP task we used a random subset of 1400 documents from DLarge and for each document $d$ from this subset retrieved the $k \mathrm{NN}$ by computing the distances to the remaining 1399 plus a comparison to itself to ensure that all metrics are executing properly. Eventually this resulted in 1960000 comparisons. In this setup $k=10$. The number of documents was chosen to simulate a situation where one has to process a query against a corpus of approximately $2 \mathrm{M}$ documents and return the top 10 results, which requires approximately the same number of comparisons. The reason not to randomly select one document and compute the distances to all other documents is that it takes a substantial amount of time to run one experiment, and lots of experiments are needed to generate statistically representative data without any biases. To tackle that we computed the same number

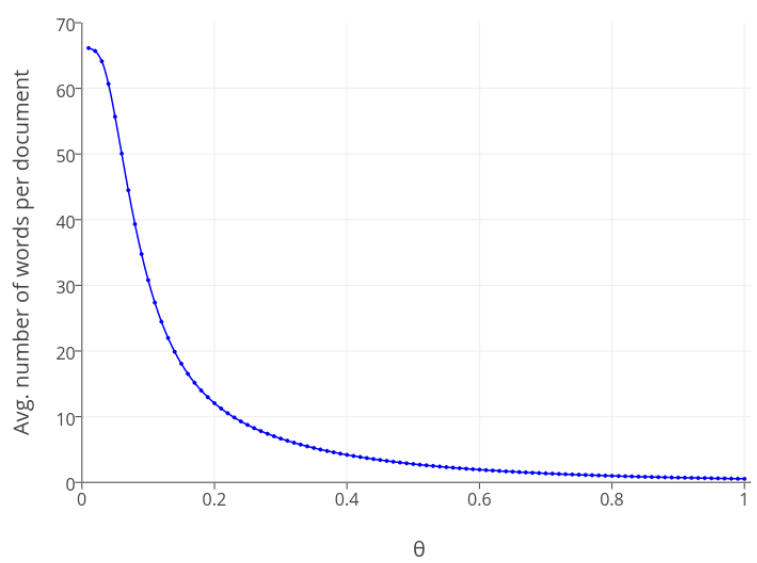

Figure 1: Average number of words selected per document based on $\theta$ 
of comparisons, which from a performance point of view imposes no limitations. And with the smaller subset and full document to document comparisons, we can analyze the results quickly and in more depth generating the necessary statistical data.

We devised two versions of the TF task, because not every $d \in$ $D r n r$ has both a non-empty $D^{+}(d)$ and a non-empty $D^{-}(d)$. The first version (TF1) used the subset of all documents $d \in D r n r$ with a non-empty $D^{+}(d)$ and a non-empty $D^{-}(d)$ (there were 216 such documents in Drnr). We generated from this data set a set of triplets $\left(d, d^{+}, d^{-}\right)$(in total 517) with the property that $d^{+} \in$ $D^{+}(d)$ and $d^{-} \in D^{-}(d)$. Then, we checked if the trained distancecomputing model yielded a smaller distance for $d, d^{+}$than for $d, d^{-}$ (Triplet comparison method as per [9]). The second version (TF2) was carried out according to the following protocol:

- Fix a threshold $\lambda$. We used the mean distance between the documents in Drnr.

- Form the collection of document pairs

$$
\left\{\left(d, d^{*}\right): d \in \operatorname{Drnr}, d^{*} \in D^{+}(d) \cup D^{-}(d)\right\} .
$$

It consisted of 3304 document pairs.

- Apply the following $\lambda$-threshold decision rule to each of the above-constructed document pairs: if the distance between $d, d^{*}$ is less than $\lambda$, then $d^{*}$ should be in $D^{+}(d)$, and otherwise should be in $D^{-}(d)$

Finally, the precision of these $\lambda$-threshold predictions is calculated and reported.

The experimental setup for the TKNN consisted of computing all the distances in DKey and getting the $k=10$ neighbours for a given document $d$ with the metric under evaluation. Then using all the labels we computed the true distance, which is made a baseline for further comparison. After that we match the $k \mathrm{NN}$ generated with the two metrics and finally report the precision as the percentage of all documents that have at least one overlapping neighbour.

This concludes the experimental setup. The next subsection presents the reported results from the conducted experiments. The focus is on precision, clock CPU time (CCPU), and wall CPU time (WCPU) for each task. Additionally we measure the memory consumption for the TTP task since it is the only more memory intensive task.

\subsection{Results}

The results are presented in the following order: first starting with the TTP task results, following with the TKNN task results and finishing with the TF tasks. The section is concluded with a short discussion and results summary.

Table 2: Results from the topic prediction task (TTP).

\begin{tabular}{crrrr}
\hline Method & CCPU & WCPU & Memory & Precision \\
\hline WMD & $7865 \mathrm{~s}$ & $49197 \mathrm{~s}$ & $13.5 \mathrm{~GB}$ & $82.79 \%$ \\
SIF & $2577 \mathrm{~s}$ & $25236 \mathrm{~s}$ & $9 \mathrm{~GB}$ & $81.29 \%$ \\
WISDM & $633 \mathrm{~s}$ & $13008 \mathrm{~s}$ & $2.5 \mathrm{~GB}$ & $80.46 \%$ \\
\hline
\end{tabular}

Table 2 shows the properties measured following the experimental setup for the TTP task. The precision measures are all within 3\% and all above $80 \%$, which means that all three methods managed to predict the topic of a given document well, based on the $k \mathrm{NN}$ rule. The times reported on a single CPU are between approximately 3.6 hours for WISDM and 13.6 hours for WMD. The memory consumption is also the lowest for WISDM (2.5GB) and the highest for WMD (13.5 GB).

Table 3: $K$-nearest neighbours task (TKNN) results.

\begin{tabular}{crrr}
\hline Method & CCPU & WCPU & Precision \\
\hline WMD & $7865 \mathrm{~s}$ & $49197 \mathrm{~s}$ & $75.38 \%$ \\
SIF & $2577 \mathrm{~s}$ & $25236 \mathrm{~s}$ & $79.13 \%$ \\
WISDM & $633 \mathrm{~s}$ & $13008 \mathrm{~s}$ & $76.20 \%$ \\
\hline
\end{tabular}

Next, Table 3 reports the results from the TKNN experiments. The $k \mathrm{NN}$ computation for each metric is being reused from the TTP task (DKey is formed from the 1400 documents used for TTP). We already demonstrated for TTP how much time is necessary to compute $2 \mathrm{M}$ comparisons for a real life $k \mathrm{NN}$ scenario. In this experiment the focus is on evaluating precision. As stated in Section 4.3 evaluating $k \mathrm{NN}$ objectively and fairly using a supervised metric is a hard task, which explains the drop of precision for all metrics. The results of WISDM are above $75 \%$ and within less than 3\% boundary for precision against the top metric, which in this case is SIF. The more significant drop in WMD precision could be explained with the fact that at the core of the baseline metric are keywords, which makes it more similar in nature to SIF and WISDM than to WMD. Also WMD relies solely on the quality of the underlying word embeddings model, whereas SIF and WISDM with their TF-IDF based schemes could compensate better for any imperfections or flaws in the word embeddings.

In Tables 4 and 5 are presented the results from the TF task.

Table 4: Results from the first filtering task (TF1).

\begin{tabular}{ccrr}
\hline Method & CCPU & WCPU & Precision \\
\hline WMD & $58.32 \mathrm{~s}$ & $89.29 \mathrm{~s}$ & $85.71 \%$ \\
SIF & $32.60 \mathrm{~s}$ & $59.51 \mathrm{~s}$ & $85.11 \%$ \\
WISDM & $17.35 \mathrm{~s}$ & $48.12 \mathrm{~s}$ & $83.33 \%$ \\
\hline
\end{tabular}

The first experiment TF1 is based on triplet comparison for the documents $d$ in Drnr that have both non empty set $D^{+}(d)$ and $D^{-}(d)$. The results show similar differences in precision keeping all results within $3 \%$. In terms of performance a consistent big gap starting from 48.12s for WISDM and ending at 89.29s for WMD can be observed. The improved precision compared to other tasks comes from the well-defined data set which predisposes for easy annotation.

Table 5: Results from the second filtering task (TF2).

\begin{tabular}{crrr}
\hline Method & CCPU & WCPU & Precision \\
\hline WMD & $446.46 \mathrm{~s}$ & $584.48 \mathrm{~s}$ & $73.25 \%$ \\
SIF & $298.77 \mathrm{~s}$ & $386.51 \mathrm{~s}$ & $80.70 \%$ \\
WISDM & $133.33 \mathrm{~s}$ & $271.57 \mathrm{~s}$ & $78.17 \%$ \\
\hline
\end{tabular}

The second experiment TF2 required more distance computations which resulted in a higher processing time but still kept the 
proportions and order of methods the same. However, the precision for this task dropped, mainly because the definition of the threshold $\lambda$ assumes that the annotators can linearly separate the documents into positive and negative data sets, which during annotation might not always hold true. Also WMD in this experiment performs the worst. A possible explanation could be the fact that the other two methods are based on correlation functions which yield results as probability of similarity. That gives a more natural threshold of around 0.5 , whereas for WMD the mean value does not have the exact same meaning.

The results reported in the previous section show a consistent speed-up of WISDM in comparison to the other models - from 20 to $50 \%$ compared to SIF on all tasks and from 45 to $75 \%$ compared to WMD. WISDM also shows better management of resources presenting possible capabilities for processing of online streams of data. But more importantly it manages to keep the margin for the precision of up to $3 \%$ from the best method, which is a really small trade-off considering the reported performance.

As a final remark, we carried out additional experiments with two other distance-computing mechanisms on the filtering task TF1. We wanted to compare WISDM with metrics that are even simpler and faster in the hope of highlighting its advantages and disadvantages. The first distance-computing mechanism represented each document as a vector of its words' TF-IDF scores (cf. Appendix A.1 of [2] and Subsection 2.4 of [15]). The second one represented each document as a TF-IDF-weighted sum of its words' word2vec representations (cf. Subsection 2.2 of [15]). In either case, the distance between two given documents was computed as the cosine similarity between the documents' vector representations. While these two additional distance-computing mechanisms required less computational time than WISDM (15 to $16 \%$ speed up with very minor difference between the two approaches), the precision achieved was $67.1 \%$ for the former and $64.3 \%$ for the latter. This precision drop is significant compared to the other three metrics. Also their precision was not high enough to be considered acceptable for the TF1 task. For that reason we did not pursue further investigation and experiments on the other tasks.

\section{CONCLUSION AND FUTURE WORK}

In this work we presented the Word importance-based similarity of documents metric (WISDM). It is an embeddings-based metric, which takes advantage of a TF-IDF model to reduce the amount of words used for computing the distance between two text documents. WISDM was evaluated on three different tasks with three different data sets, all in the context of researchers' problems when analyzing scientific articles. During the evaluation, it was compared to two state-of-the-art methods - SIF and WMD. As results demonstrate, WISDM managed to outperform them by a significant margin with only a minor compromise on precision.

For future work the authors will continue following three different directions. The first one is to formalize the mathematical framework around the complexity of the algorithm and the tuning mechanism of $\theta$. The second one is to develop a version of WISDM that can be executed on GPUs. Since WISDM is based on matrix algebra, it is reasonable to utilize GPUs at least for computationally intensive tasks (such as finding a given document's $k$ nearest neighbours). Finally an attempt will be made to improve the metric's precision without compromising the performance. One approach could be using pre-processing techniques such as lemmatization. Preliminary results already show that a $3 \%$ precision gain could be achieved at the cost of a $3 \%$ performance loss. However, more experiments have to be conducted and other necessary information need to be gathered - such as memory consumption. Another approach for improving precision could be developing a supervised extension of WISDM which will utilize (human-provided) labeling data for document distance computations.

\section{REFERENCES}

[1] Amazon. 2017. Amazon AWS EC2 Types. (2017). https://aws.amazon.com/ec2/ instance-types/, last visited 2017-04-28.

[2] Sanjeev Arora, Yingyu Liang, and Tengyu Ma. 2017. A simple but tough-tobeat baseline for sentence embeddings. In International Conference on Learning Representations. To Appear.

[3] Marco Baroni, Georgiana Dinu, and Germán Kruszewski. 2014. Don’t count, predict! A systematic comparison of context-counting vs. context-predicting semantic vectors. In Proceedings of the 52nd Annual Meeting of the Association for Computational Linguistics (Volume 1: Long Papers). Association for Computational Linguistics, Baltimore, Maryland, 238-247. https://doi.org/10.3115/v1/P14-1023

[4] Yoshua Bengio, Réjean Ducharme, Pascal Vincent, and Christian Janvin. 2003. A Neural Probabilistic Language Model. f. Mach. Learn. Res. 3 (mar 2003), 1137-1155. http://dl.acm.org/citation.cfm?id=944919.944966

[5] David M. Blei, Andrew Y. Ng, and Michael I. Jordan. 2003. Latent Dirichlet Allocation. F. Mach. Learn. Res. 3 (March 2003), 993-1022. http://dl.acm.org/ citation.cfm?id $=944919.944937$

[6] Peter F. Brown, Vincent J. Della Pietra, Robert L. Mercer, Stephen A. Della Pietra, and Jennifer C. Lai. 1992. An Estimate of an Upper Bound for the Entropy of English. Comput. Linguist. 18, 1 (March 1992), 31-40. http://dl.acm.org/citation. cfm?id=146680.146685

[7] Jonathan Chang, Sean Gerrish, Chong Wang, Jordan L. Boyd-graber, and David M. Blei. 2009. Reading Tea Leaves: How Humans Interpret Topic Models. In Advances in Neural Information Processing Systems 22, Y. Bengio, D. Schuurmans, J. D. Lafferty, C. K. I. Williams, and A. Culotta (Eds.). Curran Associates, Inc., 288-296. http://papers.nips.cc/paper/ 3700-reading-tea-leaves-how-humans-interpret-topic-models.pdf

[8] T. Cover and P. Hart. 2006. Nearest Neighbor Pattern Classification. IEEE Trans. Inf. Theor. 13, 1 (Sept. 2006), 21-27. https://doi.org/10.1109/TIT.1967.1053964

[9] Andrew M. Dai, Christopher Olah, and Quoc V. Le. 2015. Document Embedding with Paragraph Vectors. CoRR abs/1507.07998 (2015). http://arxiv.org/abs/1507. 07998

[10] Go Huang, Chuan Guo, Matt J. Kusner, Yu Sun, Killian Q. Weinberger, and Fei Sha. 2016. Supervised Word Mover's Distance. 29th Conference on Neural Information Processing Systems (2016)

[11] M. J. Kusner, Y. Sun, N. I. Kolkin, and K. Q. Weinberger. 2015. From word embeddings to document distances. ICML (2015).

[12] Quoc V. Le and Tomas Mikolov. 2014. Distributed Representations of Sentences and Documents. CoRR abs/1405.4053 (2014). http://arxiv.org/abs/1405.4053

[13] Tomas Mikolov, Kai Chen, Greg Corrado, and Jeffrey Dean. 2013. Efficient Estimation of Word Representations in Vector Space. CoRR abs/1301.3781 (2013). http://arxiv.org/abs/1301.3781

[14] Jeffrey Pennington, Richard Socher, and Christopher D. Manning. 2014. GloVe: Global Vectors for Word Representation. In Proceedings of the 2014 Conference on Empirical Methods in Natural Language Processing (EMNLP). Association for Computational Linguistics, Doha, Qatar, 1532-1543. https://doi.org/10.3115/v1/ D14-1162

[15] Marek Rei and Ronan Cummins. 2016. Sentence Similarity Measures for FineGrained Estimation of Topical Relevance in Learner Essays. CoRR abs/1606.03144 (2016). http://arxiv.org/abs/1606.03144

[16] P. Robert and Y. Escoufier. 1976. A Unifying Tool for Linear Multivariate Statistical Methods: The RV- Coefficient. Journal of the Royal Statistical Society. Series C (Applied Statistics) 25, 3 (1976), 257-265. http://www.jstor.org/stable/2347233

[17] Gerard Salton and Christopher Buckley. 1988. Term-weighting Approaches in Automatic Text Retrieval. Inf. Process. Manage. 24, 5 (Aug. 1988), 513-523. https://doi.org/10.1016/0306-4573(88)90021-0

[18] CORE Team. 2017. CORE: Portal for open access research. (2017). http://core.ac. $\mathrm{uk} /$, last visited 2017-04-28. 


\section{Appendices}

\section{A THETA EXPERIMENTS}

This appendix presents the results from hyper-tuning experiments conducted on the TF1 task with varying $\theta$ parameter for WISDM. The setup for the experiments was kept as described in Section 4.3. Figure 2 displays a 3D graph representing the relation between $\theta$, time for execution of all comparisons needed for TF1 and resulting precision. The highlighted point on the 3D line refers to the experiment of WISDM on TF1 task presented in Section 4.4. As it can be seen, when $\theta$ increases the time for execution drops due to smaller amount of words selected, but as expected the precision also drops because by selecting very few words important information could and is omitted. Furthermore with $\theta$ approaching 1, as it can be seen on Figure 1, the average words selected per document are approaching 1 as well, which makes the comparison between two documents equal to the comparison of the word vectors of two words one from each. This explains on Figure 2 the significant precision drop when $\theta$ is approaching 1. More concretely the precision on the 3D graph drops from maximum $88 \%$ at $\theta=0.3$ to $50 \%$ at $\theta=1$, where the time drops with around $20 \%$ throughout the examined range. In the future more work needs to be done to justify the processes happening in the range $[0.05,0.35]$ and describe the reasons for the drop of precision at $\theta=0.15$ and significant increase at $\theta=0.3$. The intuition is that having too many words may introduce too much noise to the comparison between the documents and reduce the precision and too few might miss important information, which means the right equilibrium has to be found and mathematically justified.

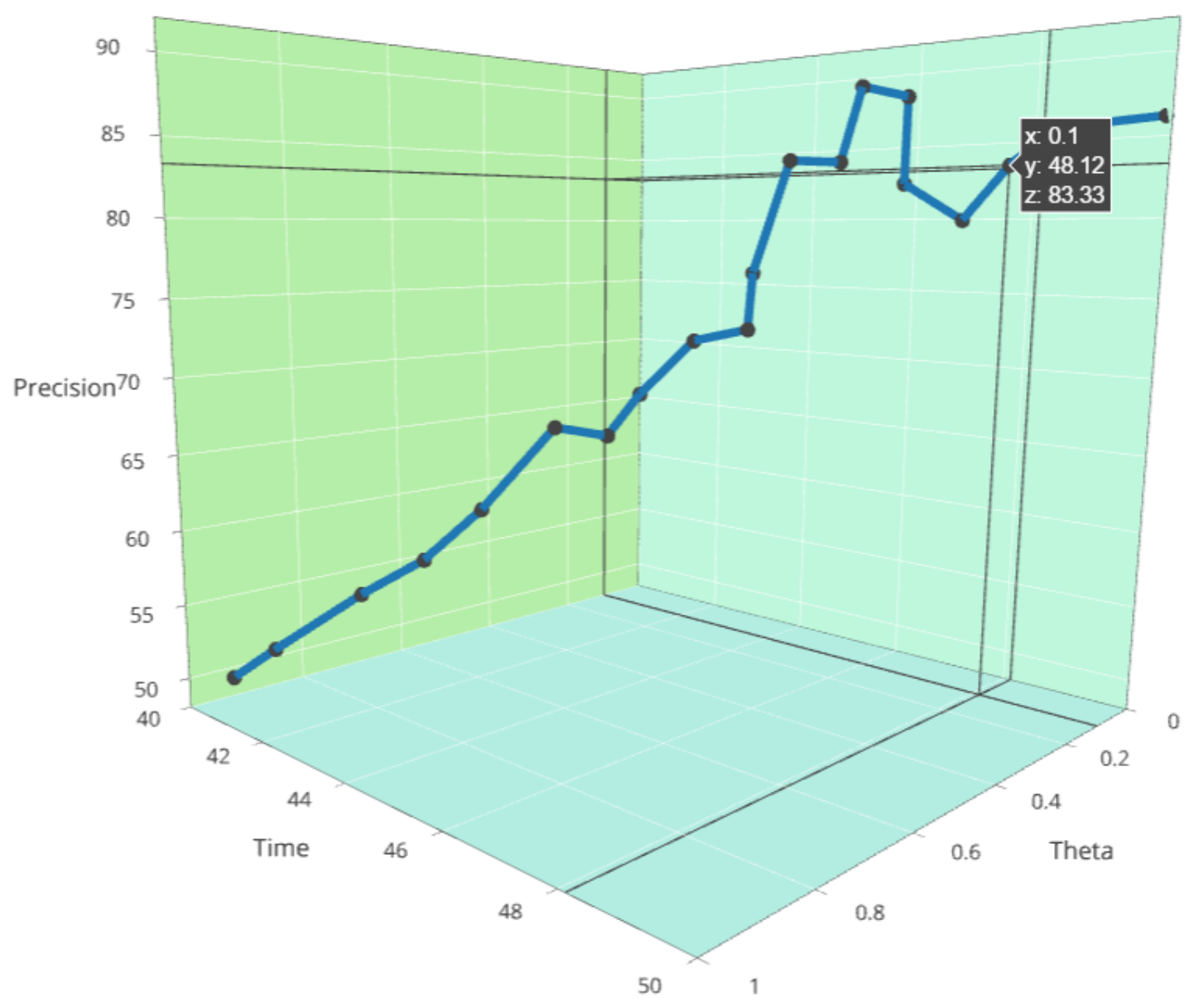

Figure 2: Precision and time change based on $\theta$ (TF1 task) 